discordance between IHC and ISH (single probe silver ISH, SISH; or dual probe fluorescence ISH, FISH). Of the 1,701 HER2 IHC 0 test results retrieved, $99.2 \%$ were HER2 SISH negative, $0.8 \%$ equivocal, and no SISH positive cases were identified. Of the IHC 0 and SISH equivocal cases, all were subsequently shown to be HER2 negative by further FISH testing. Of the 881 IHC $1+$ test results, $96.7 \%$ were SISH negative, $3.0 \%$ equivocal, and $0.3 \%$ were positive. The majority of IHC $1+$ cases with equivocal or positive SISH were ultimately shown to be HER2 negative by FISH. The IHC $1+$ cases that were subsequently shown to be HER2 amplified by FISH were reviewed and some exhibited either tumour heterogeneity or were in the neoadjuvant setting. Our review supports the current recommendation against reflexive ISH testing of IHC 0 and $1+$ cases, and referral for further testing in cases of tumour heterogeneity.

\section{ANALYSIS OF PTEN EXPRESSION IN PROSTATE CANCER}

Jeremy N. Pulvers $^{1}$, Anthony J. Gill ${ }^{1,2}$

${ }^{1}$ NSW Health Pathology, Department of Anatomical Pathology, Royal North Shore Hospital, St Leonards NSW, Australia; and ${ }^{2}$ Sydney Medical School, University of Sydney, Sydney, NSW, Australia

Mutations in the tumour suppressor gene PTEN have been identified in a variety of cancers, including thyroid, breast, kidney and endometrial carcinoma. PTEN loss identified by immunohistochemistry (IHC) in prostate cancer has been associated with higher Gleason score, early recurrence, castrate resistance, metastases, and shorter survival. Here we performed PTEN IHC on a tissue microarray (TMA) and analysed 288 prostate adenocarcinoma cases. 20\% of cases showed PTEN loss. PTEN negative cases showed a higher Gleason score and Grade group, however among Gleason 7 cases, PTEN loss was not associated with a significant increase in Group 3 verses Group 2. PTEN negativity was associated with adverse tumour features including perineural and lymphovascular invasion, however this was no longer significant when cases were matched by Gleason score. PTEN loss by IHC appears to be a promising biomarker of aggressive prostate carcinoma. However further research is required into the role of PTEN in prostate cancer.

\section{NESTED VARIANT OF UROTHELIAL CARCINOMA CAN BE CONFUSED WITH BENIGN TUMOURS SUCH AS INVERTED PAPILLOMA}

\section{Romina Rabbani, Moammar Alshimirti \\ Anatomical Pathology Department, Wollongong Hospital, Wollongong, NSW, Australia}

Nested variant of urothelial carcinoma is a rare, cytologically bland variant of invasive urothelial carcinoma that is histologically characterised by disorderly proliferation of discrete to confluent irregular crowded large numbers of small nests beneath the urothelium.

The major problem is the distinction between nested variant of urothelial carcinoma and the benign proliferative lesions of urothelium, such as inverted papilloma, von Brunn nests, cystitis cystica, cystitis glandularis, nephrogenic adenoma, and paraganglioma.
The bland appearance of the cells can give a misleading impression and the tumours are sometimes misdiagnosed as benign lesions, leading in some cases to a significant delay in concluding the correct diagnosis and therefore diagnosed at higher stage.

We review the case of a 76-year-old male, with low grade papillary urothelial carcinoma associated with nested variant of urothelial carcinoma.

\section{IS TISSUE THE ISSUE? OPTIMISING THE REFERRAL PATHWAY OF GLIOMAS FOR CGH-SNP ARRAY}

Lauren Rimmer ${ }^{1}$, Nigel Maher ${ }^{1}$, Anna Stroud ${ }^{1}$, Julia Low ${ }^{1}$, Michael Rodriguez ${ }^{1}$, Farida Zabih ${ }^{2}$, Robyn Lukeis ${ }^{2}$, Peter Earls ${ }^{1}$ Department of Anatomical Pathology and Molecular Oncology, SydPath, St Vincent's Hospital, Sydney, Australia; and ${ }^{2}$ Department of Cancer Genetics, SydPath, St Vincent's Hospital, Sydney, Australia

Background: cIMPACT-NOW guidelines state optimal assessment of WHO grade II/III gliomas requires analysis of copy number variations (CNVs). SydPath has pioneered a referral pathway for affordable, validated comparative genomic hybridisation single nucleotide polymorphism (CGH-SNP) array for $\mathrm{CNV}$ analysis of gliomas.

Aim: To optimise the referral pathway of gliomas for CGH-SNP array, enabling scalability to external centres.

Methods: Consecutive adult patients with glial neoplasms received at SydPath over two years to June 2019 were retrospectively analysed. Referral processes and outcomes of CGHSNP array were recorded.

Results: Of 77 patients with glial neoplasms, six IDH-wildtype gliomas with grade II/III morphology were upgraded to grade IV following CNV analysis as per cIMPACT-NOW guidelines. Identification of high-grade morphology on subsequent histological examination enabled timely cancellation of four tests. Ten cases with available tissue were not referred, all prior to cIMPACT-NOW guideline publication. Thirteen cases lacked tissue for referral; only four tests failed due to insufficient tissue.

Discussion: Referral for CGH-SNP array provided clinically relevant $\mathrm{CNV}$ analysis of gliomas. Improved efficiency can be achieved with our optimised tissue and case selection criteria, including complete morphological and IDH status assessment prior to testing. This will enable referral from external centres, allowing widespread adoption of cIMPACT-NOW guidelines using an affordable, validated test.

\section{References}

1. Brat DJ, Aldape K, Colman H, et al. cIMPACT-NOW update 3: recommended diagnostic criteria for "Diffuse astrocytic glioma, IDH-wildtype, with molecular features of glioblastoma, WHO grade IV". Acta Neuropathol 2018; 136: 805-10.

2. Wood MD, Halfpenny AM, Moore SR. Applications of molecular neuro-oncology - a review of diffuse glioma integrated diagnosis and emerging molecular entities. Diagn Pathol 2019; 14: 29-44.

\section{EXTRANODAL ROSAI-DORFMAN DISEASE, A RARE DIAGNOSIS IN SOFT TISSUE PATHOLOGY}

Nicholas C. Roetger, Claire Chambers

Anatomical Pathology Department, Sullivan Nicolaides

Pathology, Bowen Hills, Qld, Australia 